\title{
Volumetric assessment of lymph node metastases in patients with non-seminomatous germ cell tumours treated with chemotherapy
}

\author{
Carlos I. Basilio-de-Leo, MD; ' Christian I. Villeda-Sandoval, MD; Carolina Culebro-García, MD; ${ }^{+}$ \\ Francisco Rodríguez-Covarrubias, MD; Ricardo A. Castillejos-Molina, MD*
}

*Department of Urology, Instituto Nacional de Ciencias Médicas y Nutrición Salvador Zubirán, Mexico City, Mexico; †'Department of Radiology, Instituto Nacional de Ciencias Médicas y Nutrición Salvador Zubirán, Mexico City, Mexico

Cite as: Can Urol Assoc J 2015;9(5-6):E247-51. http://dx.doi.org/10.5489/cuai.2152

Published online May 13, 2015.

\section{Abstract}

Introduction: We evaluate volumetry and RECIST (Response Evaluation Criteria In Solid Tumors) as methodologies for response after chemotherapy for non-seminomatous germ cell tumour with retroperitoneal lymph node metastases.

Methods: We performed a retrospective analysis of non-seminomatous testicular tumours and concurrent retroperitoneal lymph node metastases, which received chemotherapy and had computed tomography scans before and after treatment. Volumetric analysis and RECIST criteria were used to calculate response rates. We included a new category (favourable response) for patients with response rates between $<100 \%$ and $>70 \%$. We calculated the correlation between volumetric and RECIST criteria with histological and clinical variables.

Results: In total, 18 patients met the inclusion criteria. Histopathologic analysis of orchiectomy showed teratoma in $55.5 \%$ of patients, and those without teratoma had predominantly embryonal carcinoma. The mean baseline volume of retroperitoneal metastases was $447 \mathrm{cc}$, the mean post-chemotherapy volume was $33.6 \mathrm{cc}$, and the response rate was $62.6 \%$. According to RECIST criteria, the mean baseline diameter was $4.93 \mathrm{~cm}$, the mean post-chemotherapy diameter was $2.39 \mathrm{~cm}$, and the response rate was $42.4 \%$. Large post-chemotherapy residual masses correlated in both classifications with teratoma. The response rate was associated with the need for surgical treatment and the volumetric classification correlated with the need for lymphadenectomy.

Conclusions: This study evaluated volumetry as a way to measure clinical response in lymph node metastases of non-seminomatous germ cell tumours. Volumetric analysis is the next step in the evaluation of response rate; its accuracy remains to be determined. Teratoma had greater residual masses and our classification correlated with the need for lymphadenectomy.

\section{Introduction}

Testicular cancer is the most common malignancy among men aged between 20 and 40. It represents about $1 \%$ of male neoplasms and 5\% of urological tumours. Germ cell tumours (GCT) can be divided in two groups: seminomatous and non-seminomatous germ cell tumour (NSGCT). At diagnosis, regional or distant metastases are present in about two-thirds of NSGCTs. ${ }^{1-4}$

The retroperitoneum is the initial site of metastases in $70 \%$ to $80 \%$ of patients with GCT, disseminated via lymphatic channels from the primary tumour. Imaging plays a major role in the initial diagnosis, but it is also an integral part for response assessment and follow-up after systemic therapy. ${ }^{5,6}$

Abdominopelvic computed tomography (CT) scan is the standard method for analyzing response to treatment. A CT scan offers a sensitivity of $70 \%$ to $80 \%$ in determining the state of the retroperitoneal nodes; ${ }^{6}$ a CT scan uses a 3-mm threshold to define metastatic nodes in the lymphatic landing zones. In clinical stages I and II, the understaging rate is $25 \%$ to $30 \% .^{7}$ After chemotherapy, a residual nodal mass may remain. Residual masses should be measured as a therapeutic response to select ideal candidates for further treatment or surveillance. ${ }^{8}$

Response has been assessed using the RECIST criteria (Response Evaluation Criteria in Solid Tumours) in clinical trials, ${ }^{6}$ whereas the World Health Organization (WHO) criteria $^{5}$ are used in daily practice. Volumetric analysis of lymph nodes may be a promising measurement method and has been successfully introduced for lung and liver lesions. ${ }^{9}$

We evaluate volumetric and unidimensional measurements to assess retroperitoneal lymph node metastases of NSGCTs in patients treated with chemotherapy, and compare these results. 


\section{Methods}

\section{Patient selection}

This is a retrospective cross-sectional study of a single-centre testicular tumour prospective database, from 2005 to 2012. Patients diagnosed with NSGCT and with retroperitoneal lymph node metastases with or without distant metastases were selected. We included those with orchiectomy performed at our centre, and treated with BEP (bleomycin, etoposide and cisplatinum) chemotherapy. All patients had pre- and post-chemotherapy $\mathrm{CT}$ imaging.

We collected demographic variables (age), diagnostic examination results (imaging studies), tumour markers (alphafetoprotein, beta-human chorionic gonadotropin, lactate dehydrogenase), clinical staging information, treatment information (orchiectomy, chemotherapy, retroperitoneal lymph node dissection) and tumour characteristics (histopathology).

CT scanning of the abdomen was performed (Siemens Somatom Sensation 64, Siemens Medical Solutions and General Electric Lightspeed VCT64, Malvern, PA). Patients received a weight-adapted dose of intravenous non-ionic contrast media, iopromide $300 \mathrm{mg} / \mathrm{mL}$ (Ultravist 300, Bayer). The acquisition was in the craniocaudal direction at 80 seconds of contrast administration in the venuos phase with slice thickness of $3 \mathrm{~mm}$. The images were analyzed with the Advantage Workstation 4.2 for diagnostic imaging (General Electric Healthcare).

Each slice of target lesion was evaluated, the contour of the lesion was delimited avoiding vascular structures of the retroperitoneum, the volume was calculated with volumetric tool, and area of all slices were integrated (Fig. 1, Fig. 2). When more than one target lesion presented in a patient, we obtained a sum of volumes.

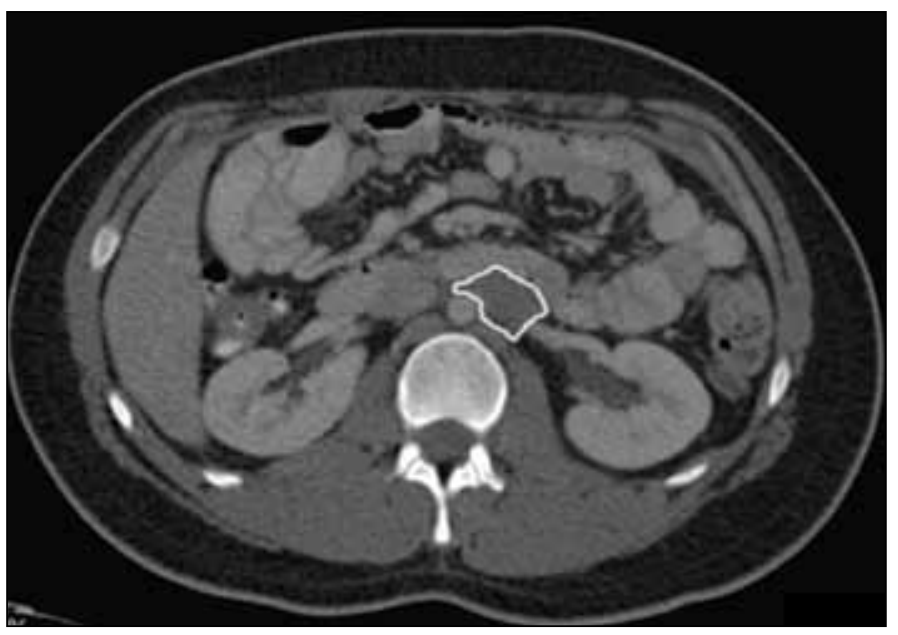

Fig. 1. Segmentaion of para-aortal lymph node.
Diameter measurements were quantified manually in a representative slice, and a short axis was obtained according to RECIST 1.1 on axial slices. When more than one target lesion was present, a sum of diameters were calculated and reported as the sum diameters.

Volumetric analysis and RECIST criteria were used to calculate response rates (difference in tumour burden from the initial to the post-treatment CT scan, expressed in percentage). Then, the response for unidimensional method was categorized according to the RECIST 1.1 criteria into one of the following:

- Complete response (CR): disappearance of all target lesions, lymph nodes must have reduction in short axis to $<10 \mathrm{~mm}$;

- Partial response (PR): at least 30\% decrease in the sum of diameters of target lesions;

- Progressive disease (PD): at least $20 \%$ increase in the sum of diameters of target lesions; and

- Stable disease (SD): qualify between PR and PD.

To be considered pathologically enlarged and measurable, a lymph node must be $\geq 15 \mathrm{~mm}$ in short axis. Only short axis will be measured and followed, and will contribute to the baseline sum.

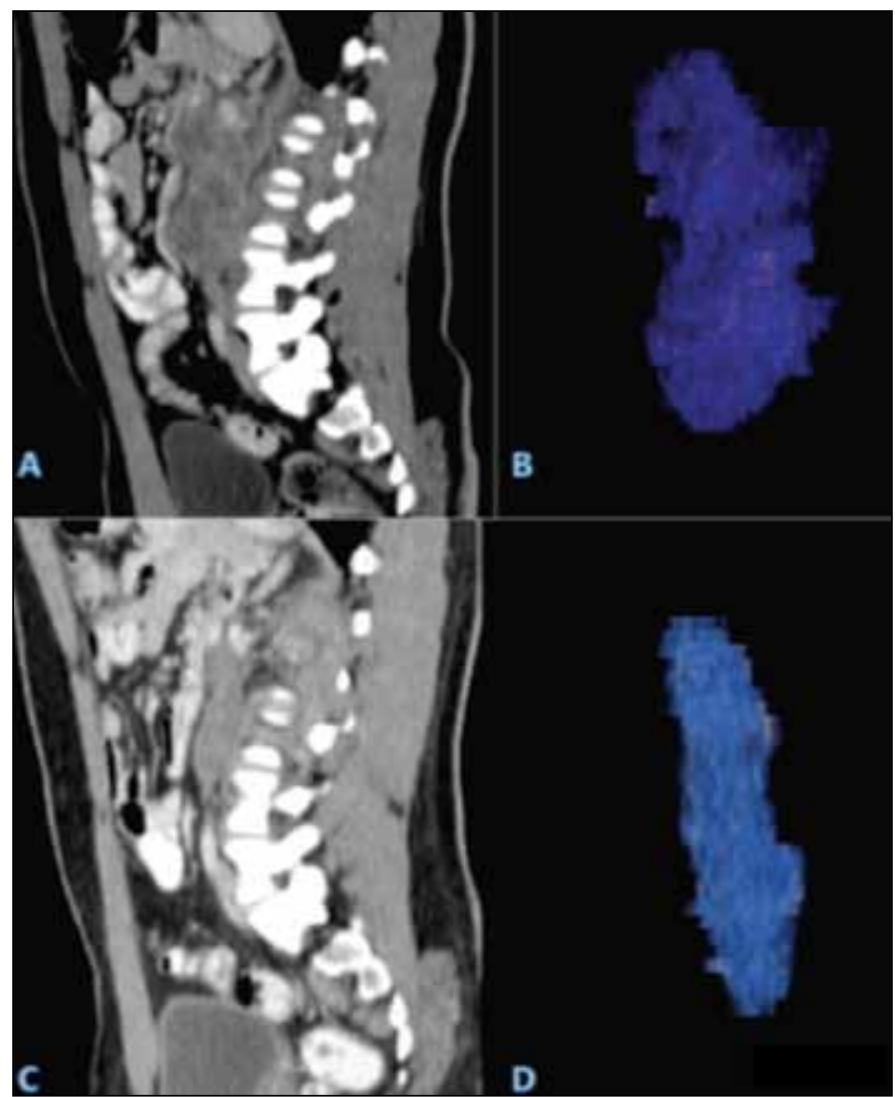

Fig. 2. A) and C) Multidetector computed tomography sagittal reconstruction Para-aortal lymph node before and after chemotherapy, respectively. B) and D) Volumetry pre- and post-chemotherapy (response rate of $80 \%$ ). 
We added a category between CR and PR - favourable response (FR), which included response rates between $<100 \%$ and $>70 \%$. The volumetric analysis response was categorized according this classification.

- CR: disappearance of all target lesions, lymph nodes must have reduction in short axis to $<10 \mathrm{~mm}$ );

- FR: response between $<100 \%$ and $\geq 70 \%$;

- PR: $\geq 30 \%$ and $<70 \%$ decrease in the sum of diameters of target lesions;

- PD: at least $20 \%$ increase in the sum of diameters of target lesions; and

- SD: qualify between PR and PD.

Statistical analyses were performed using Statistical Product and Service Solutions (SPSS) version 17.0 (Chicago, IL). Correlation analysis was performed between clinical, histological, and response methods assessments. Statistical significance was set at $p<0.05$.

\section{Results}

In total, 18 patients met the inclusion criteria, all of them were diagnosed with NSGCT. The mean age was 26.2 (range: 19-43), with most patients having right-side tumours. Baseline staging (TNM) showed distant metastatic disease in almost three-quarters of patients. Retroperitoneal lymph node metastases were divided in three categories based on their dimensions: $<2 \mathrm{~cm}, 2-5 \mathrm{~cm},>5 \mathrm{~cm})$. Orchiectomy specimens were analyzed by an expert pathologist and divided by the presence of teratoma. The mean follow-up was 47 months (Table 1).

Patients received chemotherapy consisting of 3 to 4 cycles of cisplatin, etoposide and bleomycin (PEB) (cisplatin

\begin{tabular}{lc}
\hline \multicolumn{2}{l}{ Table 1. Clinical-pathological characteristics } \\
\hline Age, years & 26.2 (range: $19-43)$ \\
Primary testicular tumour side & \\
Left & $7(38.9 \%)$ \\
Right & $11(61.1 \%)$ \\
Stage & \\
Ila & 1 \\
Ilb & 2 \\
Ilc & 1 \\
Illa & 2 \\
IIlb & 9 \\
IIIc & 3 \\
Lymph node size, cm & \\
$<2$ & $11.1 \%(2 / 18)$ \\
$2-5$ & $50 \%(9 / 18)$ \\
$>5$ & $38.9 \%(7 / 18)$ \\
Orchiectomy histology & \\
Teratoma & $55.5 \%(10 / 18)$ \\
Non-teratoma & $44.5 \%(8 / 18)$ \\
Embryonal carcinoma & $33.3 \%(6 / 18)$ \\
Seminoma & $16.6 \%(3 / 18)$ \\
Yolk sac & $11.1 \%(2 / 18)$ \\
\hline
\end{tabular}

$20 \mathrm{mg} / \mathrm{m}^{2}$ on days 1 to 5 , etoposide $100 \mathrm{mg} / \mathrm{m}^{2}$ on days 1 to 5 , bleomycin $30 \mathrm{mg}$ on days 1,8 and 15) and were reevaluated. Volumetric and unidimensional measurements were obtained before and after chemotherapy, and response rates were calculated for each method (Table 2).

Three patients did not require a second treatment. Fifteen patients required post-chemotherapy surgery according to the presence of a residual mass $(\geq 1 \mathrm{~cm})$, the mean volume was $33.6 \mathrm{~cm}^{3}$ and the mean linear measurement was $2.39 \mathrm{~cm}$. Post-lymphadenectomy residual masses were sent to pathology and analyzed: $46.6 \%(7 / 15)$ presented with necrosis, $33.3 \%$ (5/15) with teratoma, and 20\% (3/15) with viable tumour. The response rate and residual mass, measured by volumetry in the presence of necrosis, were $49.3 \%$ and $40.1 \mathrm{~cm}^{3}$ versus $63.8 \%$ and $40.2 \mathrm{~cm}^{3}$ in the presence of a teratoma-viable tumour, respectively. When RECIST criteria were used, the response rate and residual mass in the presence of necrosis were $31.5 \%$ and $2.7 \mathrm{~cm}$ versus $40.7 \%$ and $2.8 \mathrm{~cm}$ in a teratoma-viable tumour; these differences were not statistically significant.

Patients were divided in two groups depending on the presence of a teratoma (on histology of primary tumour or lymphadenectomy residual mass), and we observed correlation in both classifications between the persistence of a residual mass and the presence of teratoma. Patients with teratoma had a greater tumour burden (Table 3).

Metastases with a worse response rate to chemotherapy needed lymphadenectomy, assessed by both methods (volumetry $p=0.033$, RECIST $p=0.023$ ). Then, we categorized patients according to RECIST criteria when unidimensional method was used, and we proposed a classification adding a category of "favourable response" when volumetric measurement was used (Table 4). The response rate by categories was associated with the need of a second treatment (lymphadenectomy), meaning that patients with complete and favourable response were less likely to require surgery $(p=0.043)$, whereas those categorized by RECIST did not show that relation with surgery $(p=0.063)$.

\section{Discussion}

Metastases are commonly found in the retroperitoneum. An abdominopelvic CT scan is the most effective imaging study

Table 2. Response by volumetric and unidimensional measurements

\begin{tabular}{lcc}
\hline & Volumetry & RECIST \\
\hline \multirow{2}{*}{ Baseline } & $424.2 \pm 1350 \mathrm{cc}$ & $4.93 \pm 4.01 \mathrm{cc}$ \\
& (range: $4.09-5965)$ & (range: $1.5-18.5$ ) \\
Post-chemotherapy & $31.88 \pm 47 \mathrm{cc}$ & $2.29 \pm 1.48 \mathrm{cc}$ \\
& (range: $0-193$ ) & (range: $0-6.01$ ) \\
\multirow{2}{*}{ Response rate } & $63.6 \pm 40.46 \%$ & $42.8 \pm 28 \%$ \\
& (range: $-57.3 \%-100 \%)$ & (range: $-20 \%-100 \%$ ) \\
\hline RECIST: Response Evaluation Criteria In Solid Tumors. &
\end{tabular}




\begin{tabular}{lcc}
\hline \multicolumn{3}{l}{ Table 3. Teratoma and residual mass } \\
\hline & Volumetry (cc) & RECIST (cm) \\
\hline Teratoma & 45.94 & 3.19 \\
Non-teratoma & 18.2 & 1.38 \\
& $p=0.016$ & $p=0.002$ \\
\hline
\end{tabular}

RECIST: Response Evaluation Criteria In Solid Tumors.

for staging and follow-up after treatment. When a patient is treated with chemotherapy, it is necessary to assess for response and decide whether to provide additional treatment or surveillance. ${ }^{5}$ The decision to surgically remove a residual mass depends on the seminomatous or non-seminomatous nature of the original tumour and the size of the residual mass. ${ }^{10}$

So far, two validated criteria are accepted to assess tumour response: RECIST and WHO criteria; these are based on unidimensional and bidimensional measurements in the axial plane, respectively. These linear measurements have limitations. They miss information about the response of lymph nodes because these lesions are often presented with irregular shapes and dimension changes. Other limitations include patient-related factors (positioning, anatomy) and technical factors (slide thickness, contrast administration). ${ }^{11,12}$ Our study tries to overcome some of these issues with the use of a volumetric measurement, which has demonstrated a good correlation and accuracy with the actual size of the lesion..$^{13-15}$ Reproducibility, accessibility, and accuracy are waiting to be demonstrated in clinical practice.

We obtained volumetry by manual measurements as detailed before. In this technique one radiologist drew the boundaries of the lesion on each slide, all slides are grouped together and the volume is calculated for each lesion. Although this method is accurate, it is also time consuming and limited in its use in daily clinical practice. Nevertheless, studies have employed software and semiautomated techniques facilitating this work and volumetry measurements should be applied under expert supervision to avoid overestimation errors. ${ }^{12-18}$

The number of lesions and tumours examined for each patient depends on the response rate, and most of the recommendations are arbitrary. Usually, the number of lesions to be measured for the assessment is 2 lymph nodes and 2 targets per organ (up to 5 targets in total). The RECIST criteria suggest that 5 lesions per organ and 10 lesions in total should be measured at baseline examination, because these represent the entire tumour burden. Schwartz and colleagues showed that measuring more than 6 lesions did not yield substantial improvement. ${ }^{8}$ In fact, new RECIST criteria guidelines were developed (RECIST 1.1). These new guidelines suggest that up to 5 lesions ( 2 per organ) is enough to represent the overall tumour burden. ${ }^{19}$

In this study, we focused only on metastatic lesions present in the retroperitoneum because this location represents the

\begin{tabular}{lcc}
\hline \multicolumn{3}{l}{ Table 4. Volumetric and RECIST response classification } \\
\hline Response rate & Volumetry & RECIST \\
\hline Complete response $100 \%$ & 1 & 1 \\
Favourable response $<100 \%$ to $\geq 70 \%$ & 10 & - \\
Partial response $<70 \%$ to $\geq 30 \%$ & 5 & 12 \\
Stable disease $<30 \%$, increase $<20 \%$ & 1 & 5 \\
Progressive disease, increase $\geq 20 \%$ & 1 & 0 \\
Total & 18 & 18 \\
\hline RECIST: Response Evaluation Criteria In Solid Tumors. &
\end{tabular}

most frequently affected. Lymph node metastases are more complex, so we did not limit the number of lesions measured and analyzed measurable and suspicious lesions. In this context, volumetric analysis may be useful in overcoming the limitations associated with unidimensional or bidimensional methods. Besides the differences found in response rates between measurement methods (volumetry $62.67 \%$ vs. unidimensional $42.4 \%$ ), we also observed that some patients were re-assigned to another category according to the method used. This fact can ultimately affect further treatment decisions.

Kreuser and colleagues found a correlation between the initial and residual tumour volume, and no correlation between tumour volume and tumour markers. We analyzed the relation between residual mass and histologic features. ${ }^{20}$ Teratoma is a tumour that contains different elements of at least two of the three germ cell layers, and is usually associated with normal tumour markers. Teratomas are resistant to chemotherapy. ${ }^{1}$ Therefore, we analyzed the histologic subtype on either the initial pathology of orchiectomy or in the retroperitoneal lymph node dissection, associated with the persistence of a residual mass and the response rate. As expected, our results showed that the presence of teratoma was correlated with greater residual mass after chemotherapy due to its chemo-resistance. Response rate differences between the groups with or without teratoma were not statistically significant, although a tendency was observed - this could be overcome with more patients. Neither residual mass nor the response rate could determine the histologic findings post-lymphadenectomy.

The category criteria assigned for response, particularly PR, included a very broad range of values (for example, tumor shrinkage of $30 \%$ and $85 \%$ are considered in the same category in a lesion(s) initially measuring $10 \mathrm{~cm}$ ). Therefore, we introduced a new category between CR and PR - FR to observe if these patients have different outcomes. There was a correlation between response rate and the need of postchemotherapy surgery, suggesting that those with a favourable response ( $>70 \%$ tumour shrinkage) did not require immediate surgery.

One of the limitations of our study is its retrospective nature. It would have been helpful to include more patients. Also, manual volumetric analysis is time consuming, so a 
semi-automated technique would have been desirable if clinical applicability would have been an issue. We only included lesions present in the retroperitoneum and omitted target lesions outside the retroperitoneum. Our interest was primarily lymph nodes because they can benefit from the volumetric assessment. Another limitation is the small number of patients, although this is a pilot study. This study opens up opportunities in the study of volumetry in the metastatic field.

\section{Conclusion}

We propose volumetry as a measurement method and we evaluate the response rate for lymph node metastases of testicular tumours. Because of the limitations of linear assessment in these lesions, volumetric analysis is the next step, but its accuracy remains unknown when compared with the standard method. RECIST criteria and volumetric assessment are adequate and promising methods to assess response in lymph node metastases. So far, response rate or residual mass volume cannot predict necrosis. Our classification showed a correlation with the need of lymphadenectomy. More studies are needed to validate these findings.

Acknowledgments: Ricardo A. Castillejos Molina and Francisco Rodríguez-Covarrubias designed, reviewed and supervised the research, Carlos I. Basilio de Leo performed the research, collected data and information, and drafted the paper, Christian Villeda Sandoval analyzed data and Carolina Culebro García performed image measurements, volumetry and image reconstructions.

Competing interests: Dr. Basilio de Leo, Dr. Villeda Sandoval and Dr. Culebro-García have nothing to disclose. Dr. Rodriguez-Covarrubias reports personal fees from GSK and Ferring, outside the submitted work. Dr. Castillejos-Molina reports personal fees from Eli Lilly and GSK, outside the submitted work.

This paper has been peer-reviewed.

\section{References}

1. Stephenson AJ, Gilligan TD. Neoplasms of the Testis. Wein AJ, Kavoussi LR, Novick AC, Partin AW, editors. Campbell-Walsh Urology, vol. 1, 10th ed. Philadelphia: Saunders Elsevier; 2011:837-70.

2. La Vecchia C, Bosetti C, Lucchini F, et al. Cancer mortality in Europe, 2000-2004, and an overview of trends since 1995. Ann Oncol 2010;21:1323-60. http://dx.doi.org/10.1093/annonc/mdp530
3. Cancer Incidence in Five Continents, Vol IX. Curado MP, Edwards B, Shin R, et al. eds. IARC Scientific Publication 2007;160.

4. Engholm G, Ferlay J, Christensen N, et al. NORDCAN-a Nordic tool for cancer information, planning quality control and research. Acta Oncol 2010;49:725-36. htrp://dx.doi.org/10.3109/02841861003782017

5. Brunereau $L$, Bruyère $F$, Linassier $C$, et al. The role of imaging in staging and monitoring testicular cancer. Diagn Interv Imaging 2012;93:310-8. http://dx.doi.org/10.1016/i.diii.2012.01.014

6. Heidenreich A, Albers $\mathrm{P}$, Classen J, et al. Imaging studies in metastatic urogenital cancer patients undergoing systemic therapy: Recommendations of a multidisciplinary consensus meeting of the Association of Urological Oncology of the German Cancer Society. Urol Int 2010;85:1-10. http:// dx.doi.org/10.1159/000318985

7. Miller AB, Hoogstraten B, Staquet $M$, et al. Reporting results of cancer treatment. Cancer 1981;47:207-14. http://dx.doi.org/10.1002/1097-0142(19810101)47:1<207::AlD-CNCR2820470134>3.0.C0;2-6

8. Schwartz LH, Mazumdar M, Brown W, et al. Variability in response assessment in solid tumors: Effect of number of lesions chosen for measurement. Clin Cancer Res 2003;9:4318-23.

9. Fabel $M$, Wulff A, Heckel F, et al. Clinical lymph node staging-influence of slice thickness and reconstruction kernel on volumetry and RECIST measurements. Eur J Radiol 2012; 81: 3124-30. http://dx.doi. org/10.1016/i.eirad.2012.03.008

10. Heidenreich A, Pfister D. Retroperitoneal lymphadenectomy and resection for testicular cancer: An update on best practice. Ther Adv Urol 2012;4:187-205. http://dx.doi.org/10.1177/1756287212443170

11. Kang H, Lee HY, Lee KS, et al. Imaging-based tumor treatment response evaluation: Review of conventional, new, and emerging concepts. Korean J Radiol 2012;13:371-90. http://dx.doi.org/10.3348/ kir.2012.13.4.371

12. Goldmacher GV, Conklin J. The use of tumour volumetrics to assess response to therapy in anticancer clinical trials. Br J Clin Pharmaco/ 2012;73:846-54. http://dx.doi.org/10.1111/j.1365-2125.2012.04179.x

13. Steger S, Franco F, Sverzellati N, et al. 3D Assessment of lymph nodes vs. RECIST 1.1. Acad Radiol 2011;18:391-4. http://dx.doi.org/10.1016/i.acra.2010.11.010

14. Puesken $M$, Buerke $B$, Gerss J, et al. Prediction of lymph node manifestations in malignant lymphoma: Significant role of volumetric compared with established metric lymph node analysis in multislice computed tomography. J Comput Assist Tomogr 2010;34:564-9. http://dx.doi.org/10.1097/ RCT.0b013e3181db2901

15. Fabel $M$, Biederer J, Jochens $A$, et al. Semi-automated volumetric analysis of artificial lymph nodes in a phantom study. Eur J Radiol 201 1;80:451-7. http://dx.doi.org/10.1016/i.ejrad.2010.10.019

16. Leibovitch I, Foster RS, Kopecky KK, et al. Improved accuracy of computerized tomography based clinical staging in low stage nonseminomatous germ cell cancer using size criteria of retroperitoneal lymph nodes. J Urol 1995; 154:1759-63. http://dx.doi.org/10.1016/S0022-5347(01)66778-8

17. Swanson DA. Role of retroperitoneal lymphadenectomy (RLDN) when patients with nonseminomatous germ cell testicular tumours are at high risk of needing lymph node surgery plus chemotherapy, in Donohue JP (ed.). Lymph Node Surgery in Urology. International Society of Urology Reports. Oxford:Isis Medical Media; 1995:133-40.

18. Buerke B, Puesken $M$, Müter $S$, et al. Measurement accuracy and reproducibility of semiautomated metric and volumetric lymph node analysis in MDCT. AJR Am J Roentgenol 2010;195:979-85. http://dx.doi. org/10.2214/AJR.10.4010

19. Eisenhaver EA, Therasse P, Bogaerts J, et al. New response evaluation criteria in solid tumours: Revised RECIST guideline (version 1.1). Eur J Cancer 2009;45:228-47. http://dx.doi.org/10.1016/i. ejca.2008.10.026

20. Kreuser $E D$, Wellert $M$, Weidenmaier $W$, et at. Feasibility and relevance of tumor volumetry for stage classification and assessment of remission of germ cell tumors. Urologe A 1989;28:344-9.

Correspondence: Dr. Ricardo A Castillejos-Molina, Department of Urology, Instituto Nacional de Ciencias Médicas y Nutrición Salvador Zubirán, Vasco de Quiroga 15, Col. Sección XVI. Tlalpan 14000, Mexico City, Mexico; rcastillejos@hotmail.com 